\title{
The potential effects of dietary food and beverage intakes on the risk of kidney stone formation
}

\author{
Os efeitos potenciais da ingestão dietética de \\ alimentos e bebidas sobre o risco de \\ formação de cálculos renais
}

Mehmet Arif ICER ${ }^{1}$ iD 0000-0002-1632-7484

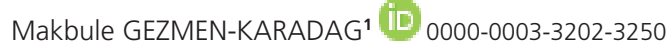

A B S T R A C T

\section{Objective}

To determine the effect of nutritional habits on kidney stone formation and recurrence.

\section{Methods}

This study was conducted on 44 healthy individuals and 44 patients diagnosed with nephrolithiasis and aging between 20 and 65 years. Participants shared their salt consumption habits, daily fluid consumption amounts and general information about themselves in a questionnaire form. In addition, food and beverage consumption frequencies of participants were recorded through a food frequency questionnaire.

\section{Results}

Salt consumption frequencies of patients are higher than that of healthy individuals in both genders $(p<0.05)$. It was found out that male individuals in the patient group salt dishes without tasting more frequently $(p<0.05)$. Daily total water consumption of both genders in patient group is lower than that of healthy individuals $(p<0.05)$. Meat consumption of male patients $(51.6 \pm 31.35 \mathrm{~g} /$ day) was found to be higher than that of healthy group $(34.1 \pm 22.58 \mathrm{~g} /$ day $)(p<0.05)$. Additionally, individuals in the patient group consume less stinging nettle, corn, plum, loquat, orange juice and lemonade than healthy individuals $(p<0.05)$.

\footnotetext{
1 Gazi University, Faculty of Health Sciences, Nutrition and Dietetics Department. 06500 Beşevler, Ankara, Turkey. Correspondence to: MA ICER. E-mail: <m.arif.icer@gmail.com>.

Article based on the dissertation thesis by MA ICER entitled "Assessment of urinary osteopontin level, dietary acid load and nutritional status of patients with kidney stone”. Gazi University; 2018.
}

\footnotetext{
How to cite this article

Icer MA, Gezmen-Karadag M. The potential effects of dietary food and beverage intakes on the risk of kidney stone formation. Rev Nutr. 2019;32:e190029. http://dx.doi.org/10.1590/1678-9865201932e190029
} 


\section{Conclusion}

Results of the study showed that total fluid intake, salt consumption habits, and vegetable, fruit and beverage consumption may be correlated with stone formation risk and nutrition habits may affect stone recurrence.

Keywords: Beverages. Feeding behavior. Food. Kidney calculi. Nutrition.

\section{RE S U M O}

\section{Objetivo}

Determinar o efeito de hábitos nutricionais na formação e recorrência de cálculos renais.

\section{Métodos}

Este estudo foi realizado em 44 individuos saudáveis e 44 pacientes com diagnóstico de nefrolitíase e envelhecimento entre 20 e 65 anos. Os participantes compartilharam seus hábitos de consumo de sal, quantidades diárias de consumo de líquidos e informações gerais sobre si mesmos em um questionário. Além disso, as frequências de consumo de alimentos e bebidas dos participantes foram registradas por meio de um questionário de frequência alimentar.

\section{Resultados}

As frequências de consumo de sal dos pacientes são maiores que as de individuos saudáveis em ambos os sexos $(p<0,05)$. Verificou-se que os indivíduos do sexo masculino no grupo de pacientes salgam os pratos sem degustar com maior frequência $(p<0,05)$. O consumo diário total de água de ambos os sexos no grupo de pacientes é menor que o dos indivíduos saudáveis $(p<0,05)$. O consumo de carne de pacientes do sexo masculino $(51,6 \pm 31,35 \mathrm{~g} / \mathrm{dia})$ foi maior que o do grupo saudável $(34,1 \pm 22,58 \mathrm{~g} / \mathrm{dia})(p<0,05)$. Além disso, indivíduos do grupo de pacientes consomem menos urtiga, milho, ameixa, nêspera, suco de laranja e limonada do que indivíduos saudáveis $(p<0,05)$.

\section{Conclusão}

Os resultados do estudo mostraram que a ingestão total de líquidos, os hábitos de consumo de sal e o consumo de vegetais, frutas e bebidas podem estar correlacionados com o risco de formação de pedra e os hábitos de nutrição podem afetar a recorrência da pedra.

Palavras-chave: Bebidas. Comportamento alimentar. Alimentos. Cálculos renais. Nutrição.

\section{NTROD U C T I O N}

Kidney stone disease is a common health problem with a multifactorial etiology arising from the interaction of metabolic, genetic and environmental factors [1]. Kidney stone disease, one of the most important preventable causes of morbidity, causes 5 billion dollars of economic loss in the United States every year [2].

Kidney stones often comprise of calcium oxalate (74\%) [3]. Stone formation is more common in men than in women [4]. Moreover, nephrolithiasis is increasing in developed and developing countries at an alarming rate [5]. Many factors such as age, gender, race, ethnicity, nutrition, daily water intake, climate, geography, physical activity and Body Mass Index (BMI) affect stone formation $[5,6]$.

There are quite a large number of studies proving the effects of nutrients on kidney stone formation [7-10]. A great majority of such studies show that nutrition has important and effective role on kidney stone formation [10-13]. It is reported that high dietary calorie, animal protein and oxalate intake and low fluid, calcium and potassium consumption are among the most important dietary risk factors in kidney stone formation [13-16]. 
Although there are a large number of studies examining the correlation between kidney stone formation and nutrition habits, data regarding which foods and beverages can reduce stone formation risk is inadequate. This study was conducted to evaluate the correlation between nutrition habits and kidney stone formation.

\section{METHODS}

This study was carried out on 44 healthy individuals and 44 healthy individuals who were adult and diagnosed with kidney stones formation by the doctors. The control group consisted of the healthy individuals. And the control group have similar gender, age and BMI with patient group. Menopausal and pregnant women were not included in this study. Besides, the individuals who have dieted in the last six months, had a disease affecting calcium metabolism, been taking the medicines which affect calcium metabolism, been taking the medicines which affect oxalate, calcium, citrate and pH levels in urine were not included in this study. The ethical committee report of the study was taken from the Ethics Committee of Clinical Investigations of Kecioren Training and Research Hospital, dated January 25, 2017 and No.2012-KAEK-15/1263. Signed informed consent was obtained from all patients and this study was conducted in accordance with the Helsinki Declaration.

Using the face-to-face interview technique, the general characteristics of the participant and the salt usage habits and the daily fluid consumption amounts of the individuals were questioned by questionnaire. Additionally, the participants answered the questions in Food Frequency Questionnaire (FFQ) for evaluation of their frequency of consumption dietary food and beverages. The researcher prepared a questionnaire of 119 articles in order to record the consumption frequency of some food and beverages under several main titles including: (a) dairy products (milk, ayran, yoghurt, feta cheese and kashar cheese); (b) meat and meat products (red meat, chicken, turkey, fish, offal, eggs, legumes and nuts); (c) vegetables (gherkin, broad bean, pumpkin, ladies finger, pea, cow pea, broccoli, brussel sprouts, dill, tomato, mallow, artichoke, basil, highbush cranberry, chicory, stinging nettle, spinach, zucchini, savoy cabbage, cauliflower, locust bean, celery, red pepper, curly lettuce, asparagus, lemon, mushroom, lettuce, parsley, corn, mint, potato, eggplant, chard, leek, sweet basil, fennel, garden rocket, cucumber, purslane, green bean, cress, radish, grape leaves, green pepper, ginger and turmeric); (d) fruits (pineapple, raspberry, pear, avocado, quince, blackberry, green almond, strawberry, mullberry, apple, plum, grapefruit, date, fig, watermelon, melon, apricot, cherry, kiwi, mandarin, mango, medlar, banana, papaya, orange, peach, grape, sour cherry and loquat); (e) beverages (tea, herbal teas, caffeinated coffee and decaffeinated coffee); (f) carbonated beverages (coke, diet coke, sparkling water, soda pop); (g) fruit juices (lemonade, grape juice, apple juice, carrot juice, orange juice, grapefruit juice, peach juice, sourcherry juice, pomegranate juice and turnip juice), and (h) alcoholic beverages (beer, malt drink, wine and other).

Qualitative data obtained from the participants were used to calculate number $(n)$ and percentage values (\%). The correlation between categorical variables was examined by means of chi-square test. Mann-Whitney $U$ test was used to compare the measurement values of two independent groups in non-parametric data on the basis of the Average $(\bar{x})$ Standard Deviation (SD) and Median $(\bar{x})$ values of qualitative data. Statistical significance rates were given between 99\% and/or 95\% Confidence Interval. Data obtained from the participants were analyzed with IBM Statistical Package for the Social Sciences 20 (SPSS Inc., Chicago, Illinois, United States). 


\section{RE S U L T S}

According to the study, there is a statistically significant correlation between salt consumption of both genders and their state of health $(p<0.05)$. It was found out that $37.9 \%$ of male patients consume their food with excessive salt, while $6.9 \%$ of healthy males do the same. In female participants, $20.0 \%$ of patients prefer excessive salt in their food; however, $6.7 \%$ of healthy individuals are fond of salty food.

It was found out that male individuals in the patient group salt dishes without tasting more frequently than healthy male individuals do $(p<0.05)$. However, such difference was not observed in female participants' salting habits ( $p>0.05$ ) (Table 1).

According to the study, there is a statistically significant correlation between total fluid intake of both genders and their state of health. Daily total fluid intake of both genders in patient group is lower than that of healthy individuals $(p<0.05)$ (Table 1$)$. It was found out that the rates of male participants who had kidney stone history in their families are $51.7 \%$, while these rates are in females $53.3 \%$ (Table 2).

There is a statistically significant difference between two genders in terms of kidney stone disease history of their fathers $(p<0.05)$ (Table 2 ). Forty percent of male patients' fathers had kidney stone disease, while $37.5 \%$ of female patients' brother or sister suffered from the disease.

Although male patients do not consume a special food for treatment of kidney stone disease, 2 female patients consume olive oil and stinging nettle to cure the disease; $37.9 \%$ of male patients consume a special beverage for treatment of the disease (highbush cranberry juice: $45.4 \%$, parsley juice: $27.3 \%$ and lemon juice: 27.3 ), while $33.3 \%$ of female patients prefer a curing beverage (highbush cranberry juice: $40 \%$, lemon juice: 20\%, kefir: 20\% and oleaster juice: $20 \%$ ). However,

Table 1. Salt use and fluid consumption status of individuals. Ankara, Turkey, 2018.

\begin{tabular}{|c|c|c|c|c|c|c|c|c|c|c|c|c|}
\hline \multirow{3}{*}{ Salt use habit/fluid intake } & \multicolumn{4}{|c|}{ Male } & \multirow{3}{*}{$\chi^{2}$} & \multirow{3}{*}{$p$} & \multicolumn{4}{|c|}{ Female } & \multirow{3}{*}{$\chi^{2}$} & \multirow{3}{*}{$p$} \\
\hline & \multicolumn{2}{|c|}{$\begin{array}{l}\text { Patient } \\
\text { Group } \\
(n=29)\end{array}$} & \multicolumn{2}{|c|}{$\begin{array}{l}\text { Control } \\
\text { Group } \\
(n=29)\end{array}$} & & & \multicolumn{2}{|c|}{$\begin{array}{l}\text { Patient } \\
\text { Group } \\
(n=15)\end{array}$} & \multicolumn{2}{|c|}{$\begin{array}{l}\text { Control } \\
\text { Group } \\
(n=15)\end{array}$} & & \\
\hline & $\mathrm{n}$ & $\%$ & $\mathrm{n}$ & $\%$ & & & $n$ & $\%$ & $\mathrm{n}$ & $\%$ & & \\
\hline Salt use in meals without salt & 5 & 17.3 & 1 & 3.4 & & & 0 & 0.0 & 1 & 6.7 & & \\
\hline Low salt & 2 & 6.9 & 4 & 13.8 & & & 0 & 0.0 & 7 & 46.6 & & \\
\hline Normal & 11 & 37.9 & 22 & 75.9 & 13.231 & $0.004^{*}$ & 12 & 80.0 & 6 & 40.0 & 11.000 & $0.012^{*}$ \\
\hline Salty & 11 & 37.9 & 2 & 6.9 & & & 3 & 20.0 & 1 & 6.7 & & \\
\hline \multicolumn{13}{|l|}{ Salting without tasting } \\
\hline Yes & 16 & 55.2 & 5 & 17.2 & \multirow{2}{*}{9.032} & \multirow{2}{*}{$0.003^{*}$} & 5 & 33.3 & 1 & 6.7 & \multirow{2}{*}{3.333} & \multirow{2}{*}{0.068} \\
\hline No & 13 & 44.8 & 24 & 82.8 & & & 10 & 66.7 & 14 & 93.3 & & \\
\hline \multicolumn{13}{|l|}{ Daily total fluid intake } \\
\hline 1000-1500mL & 4 & 13.8 & 2 & 6.9 & & & 4 & 26.7 & 1 & 6.7 & & \\
\hline 1500-2000mL & 9 & 31.0 & 3 & 10.4 & & & 2 & 13.3 & 0 & 0.0 & & \\
\hline $2000-2500 \mathrm{~mL}$ & 12 & 41.4 & 7 & 24.1 & 14.836 & $0.005^{*}$ & 5 & 33.3 & 3 & 20.0 & 10.478 & $0.033^{*}$ \\
\hline 2500-3000mL & 4 & 13.8 & 8 & 27.6 & & & 4 & 26.7 & 4 & 26.6 & & \\
\hline$\geq 3000 \mathrm{~mL}$ & 0 & 0.0 & 9 & 31.0 & & & 0 & 0.0 & 7 & 46.7 & & \\
\hline
\end{tabular}

Note: " $p<0.05$, Chi-square test was applied. More than one option has been added. 
there is not a statistically significant difference between the genders of patients and recurrence of stone formation, kidney stone history in the family, expelling stone and consumption of a special food/beverage for treatment ( $p>0.05$ ) (Table 2).

Participants were asked about consumption of 119 foods and beverages considered to be correlated with stone recurrence and the foods found to be statistically different between patient and healthy individuals were given in the Table 3.

Meat consumption of male patients $(51.6 \pm 31.35 \mathrm{~g})$ was found to be higher than that of healthy group (34.1 $\pm 22.58 \mathrm{~g})(p<0.05)$. It was found out that male patients consume less stinging nettle, locust bean, red pepper, corn and lemon than healthy males do and the difference is statistically meaningful

Table 2. General characteristics of patients. Ankara, Turkey, 2018.

\begin{tabular}{|c|c|c|c|c|c|c|}
\hline \multirow{2}{*}{ General characteristics of patients } & \multicolumn{2}{|c|}{ Male $(n=29)$} & \multicolumn{2}{|c|}{ Female $(n=15)$} & \multirow{2}{*}{$\chi^{2}$} & \multirow{2}{*}{$p$} \\
\hline & $n$ & $\%$ & $\mathrm{n}$ & $\%$ & & \\
\hline \multicolumn{7}{|l|}{ Stone formation recurrence } \\
\hline 0 & 6 & 20.7 & 3 & 20.0 & \multirow{4}{*}{0.398} & \multirow{4}{*}{0.820} \\
\hline 1 & 6 & 20.7 & 4 & 26.7 & & \\
\hline 2 & 8 & 27.6 & 3 & 20.0 & & \\
\hline$>2$ & 9 & 31.0 & 5 & 33.3 & & \\
\hline \multicolumn{7}{|l|}{ Kidney stone history in the family } \\
\hline Yes & 15 & 51.7 & 8 & 53.3 & \multirow[t]{2}{*}{0.010} & \multirow{2}{*}{0.919} \\
\hline No & 14 & 48.3 & 7 & 46.7 & & \\
\hline \multicolumn{7}{|l|}{ Kinship relation } \\
\hline Mother & 3 & 20.0 & 1 & 12.5 & \multirow{4}{*}{7.901} & \multirow{4}{*}{$0.048^{*}$} \\
\hline Father & 6 & 40.0 & 0 & 0.0 & & \\
\hline Brother or sister & 5 & 33.3 & 3 & 37.5 & & \\
\hline Uncle & 1 & 6.7 & 4 & 50.0 & & \\
\hline \multicolumn{7}{|l|}{ Passing kidney stone } \\
\hline Yes & 19 & 65.5 & 8 & 53.3 & \multirow{2}{*}{0.619} & \multirow{2}{*}{0.431} \\
\hline No & 10 & 34.5 & 7 & 46.7 & & \\
\hline \multicolumn{7}{|c|}{$\begin{array}{l}\text { Consumption of special food/beverage for kidney } \\
\text { stone extraction }\end{array}$} \\
\hline Yes & 8 & 27.6 & 5 & 33.3 & \multirow{2}{*}{0.157} & \multirow{2}{*}{0.692} \\
\hline No & 21 & 72.4 & 10 & 66.7 & & \\
\hline \multicolumn{7}{|l|}{ The type of food consumed } \\
\hline Olive oil & 0 & 0.0 & 1 & 50.0 & & \multirow{2}{*}{ - } \\
\hline Stinging nettle & 0 & 0.0 & 1 & 50.0 & & \\
\hline \multicolumn{7}{|l|}{ Type of consumed beverage } \\
\hline Parsley juice & 3 & 27.3 & 0 & 0.0 & & \multirow{5}{*}{-} \\
\hline Highbush cranberry juice & 5 & 45.4 & 2 & 40.0 & & \\
\hline Lemon juice & 3 & 27.3 & 2 & 20.0 & & \\
\hline Kefir & 0 & 0.0 & 1 & 20.0 & & \\
\hline Oleaster juice & 0 & 0.0 & 1 & 20.0 & & \\
\hline
\end{tabular}

Note: ${ }^{*} p<0.05$, Chi-square test was applied. More than one option has been added.

$(-)$ : It has not been evaluated due to the inadequate number of participants in the groups. 
Table 3. Consumption amounts of some food and beverages associated with stone formation. Ankara, Turkey, 2018.

\begin{tabular}{|c|c|c|c|c|c|c|}
\hline \multirow{3}{*}{ Food and Beverages } & \multicolumn{2}{|c|}{ Male } & \multirow{3}{*}{$Z / p$} & \multicolumn{2}{|c|}{ Female } & \multirow{3}{*}{$Z / p$} \\
\hline & $\begin{array}{l}\text { Patient Group } \\
\qquad(n=29)\end{array}$ & $\begin{array}{l}\text { Control Group } \\
\qquad(n=29)\end{array}$ & & $\begin{array}{l}\text { Patient Group } \\
\qquad(n=15)\end{array}$ & $\begin{array}{c}\text { Control Group } \\
\qquad(n=15)\end{array}$ & \\
\hline & $\bar{x}($ Min-Max $)$ & $\bar{x}($ Min-Max $)$ & & $\bar{x}($ Min-Max $)$ & $\bar{x}($ Min-Max $)$ & \\
\hline \multirow[t]{2}{*}{ Red meat } & 42.8 & 28.5 & $Z=-2.596$ & 28.5 & 28.5 & $Z=-0.021$ \\
\hline & $(6.6-142.0)$ & $(3.3-85.7)$ & $p=0.009^{*}$ & $(0-85.7)$ & $(13.3-57.1)$ & $p=0.983$ \\
\hline \multirow[t]{2}{*}{ Fish } & 13.3 & 6.7 & $Z=-1.635$ & 6.7 & 13.3 & $Z=-2.069$ \\
\hline & $(0-85.7)$ & $(0-85.7)$ & $p=0.102$ & $(0-57.1)$ & $(6.7-57.1)$ & $p=0.039^{*}$ \\
\hline \multirow[t]{2}{*}{ Brussel sprouts } & 0.0 & 0.0 & $Z=-0.951$ & 0.0 & 5.0 & $Z=-3.046$ \\
\hline & $(0-5.0)$ & $(0-10.0)$ & $p=0.342$ & $(0-5.0)$ & $(0-5.0)$ & $p=0.002^{*}$ \\
\hline \multirow[t]{2}{*}{ Dill } & 0.2 & 0.3 & $Z=-1.717$ & 0.2 & 0.3 & $Z=-2.775$ \\
\hline & $(0-2.9)$ & $(0-5.0)$ & $p=0.086$ & $(0-2.9)$ & $(0.2-2.9)$ & $p=0.006^{*}$ \\
\hline \multirow[t]{2}{*}{ Mallow } & 0.0 & 0.0 & $Z=-1.678$ & 0.0 & 2.0 & $Z=-2.945$ \\
\hline & $(0-10.0)$ & $(0-10.0)$ & $p=0.093$ & $(0-0)$ & $(0-10.0)$ & $p=0.003^{*}$ \\
\hline \multirow[t]{2}{*}{ Artichoke } & 0.0 & 0.0 & $Z=-0.229$ & 0.0 & 2.0 & $Z=-2.950$ \\
\hline & $(0-42.8)$ & $(0-10.0)$ & $p=0.819$ & $(0-0)$ & $(0-10.0)$ & $p=0.003^{*}$ \\
\hline \multirow[t]{2}{*}{ Stinging nettle } & 0.0 & 1.0 & $Z=-2.010$ & 0.0 & 5.0 & $Z=-3.230$ \\
\hline & $(0-5.0)$ & $(0-10.0)$ & $p=0.044^{*}$ & $(0-0)$ & $(0-10.0)$ & $p=0.001^{*}$ \\
\hline \multirow[t]{2}{*}{ Savoy cabbage } & 3.3 & 3.3 & $Z=-0.213$ & 0.0 & 6.7 & $Z=-2.562$ \\
\hline & $(0-28.5)$ & $(0-57.1)$ & $p=0.831$ & $(0-28.5)$ & $(0-28.5)$ & $p=0.010^{*}$ \\
\hline \multirow[t]{2}{*}{ Locust bean } & 0.0 & 1.5 & $Z=-2.624$ & 0.0 & 0.0 & $Z=-1.792$ \\
\hline & $(0-1.7)$ & $(0-14.2)$ & $p=0.009^{*}$ & $(0-14.3)$ & $(0-0)$ & $p=0.073$ \\
\hline \multirow[t]{2}{*}{ Celery } & 0.0 & 0.0 & $Z=-0.416$ & 0.0 & 5.0 & $Z=-2.456$ \\
\hline & $(0-10.0)$ & $(0-10.0)$ & $p=0.677$ & $(0-10.0)$ & $(0-42.8)$ & $p=0.014^{*}$ \\
\hline \multirow[t]{2}{*}{ Red pepper } & 0.0 & 6.1 & $Z=-2.140$ & 3.1 & 6.1 & $Z=-0.802$ \\
\hline & $(0-52.5)$ & $(0-92.0)$ & $p=0.032^{*}$ & $(0-52.5)$ & $(0-52.6)$ & $p=0.422$ \\
\hline \multirow[t]{2}{*}{ Curly lettuce } & 5.7 & 5.7 & $Z=-1.571$ & 11.4 & 5.7 & $Z=-2.917$ \\
\hline & $(0-20.0)$ & $(0-92.0)$ & $p=0.116$ & $(1.3-20.0)$ & $(0.7-92.0)$ & $p=0.004^{*}$ \\
\hline \multirow[t]{2}{*}{ Lemon } & 4.7 & 35.0 & $Z=-4.033$ & 20.0 & 35.0 & $Z=-1.949$ \\
\hline & $(0-70.0)$ & $(4.7-70.0)$ & $p=0.000^{* *}$ & $(0-70.0)$ & $(1.2-40.0)$ & $p=0.051$ \\
\hline \multirow[t]{2}{*}{ Corn } & 0.0 & 10.0 & $Z=-2.038$ & 0.0 & 6.7 & $Z=-2.258$ \\
\hline & $(0-13.3)$ & $(0-57.1)$ & $p=0.042^{*}$ & $(0-114.3)$ & $(0-57.1)$ & $p=0.024^{*}$ \\
\hline \multirow[t]{2}{*}{ Chard } & 0.0 & 0.0 & $Z=-0.067$ & 0.0 & 5.0 & $Z=-2.080$ \\
\hline & $(0-85.7)$ & $(0-42.8)$ & $p=0.947$ & $(0-10.0)$ & $(0-10.0)$ & $p=0.038^{*}$ \\
\hline \multirow[t]{2}{*}{ Pineapple } & 0.0 & 1.0 & $Z=-2.324$ & 0.0 & 0.0 & $Z=-1.152$ \\
\hline & $(0-3.2)$ & $(0-3.2)$ & $p=0.020^{*}$ & $(0-27.1)$ & $(0-3.2)$ & $p=0.249$ \\
\hline \multirow[t]{2}{*}{ Green almond } & 0.0 & 3.3 & $Z=-3.048$ & 0.0 & 0.0 & $Z=-0.075$ \\
\hline & $(0-6.7)$ & $(0-57.1)$ & $p=0.002^{*}$ & $(0-28.6)$ & $(0-28.5)$ & $p=0.940$ \\
\hline \multirow[t]{2}{*}{ Plum } & 2.0 & 6.0 & $Z=-2.419$ & 2.0 & 4.0 & $Z=-2.962$ \\
\hline & $(0-17.1)$ & $(0-34.2)$ & $p=0.016^{*}$ & $(0-17.1)$ & $(0-17.1)$ & $p=0.003^{*}$ \\
\hline \multirow[t]{2}{*}{ Date } & 0.0 & 3.3 & $Z=-3.910$ & 0.0 & 0.0 & $Z=-0.229$ \\
\hline & $(0-3.3)$ & $(0-6.7)$ & $p=0.000^{*}$ & $(0-56.6)$ & $(0-28.5)$ & $p=0.819$ \\
\hline
\end{tabular}


Table 3. Consumption amounts of some food and beverages associated with stone formation. Ankara, Turkey, 2018.

\begin{tabular}{|c|c|c|c|c|c|c|}
\hline \multirow{3}{*}{ Food and Beverages } & \multicolumn{2}{|c|}{ Male } & \multirow{3}{*}{ Z/p } & \multicolumn{2}{|c|}{ Female } & \multirow{3}{*}{$Z / p$} \\
\hline & $\begin{array}{l}\text { Patient Group } \\
\qquad(\mathrm{n}=29)\end{array}$ & $\begin{array}{l}\text { Control Group } \\
\qquad(n=29)\end{array}$ & & $\begin{array}{l}\text { Patient Group } \\
\qquad(n=15)\end{array}$ & $\begin{array}{c}\text { Control Group } \\
\quad(n=15)\end{array}$ & \\
\hline & $\bar{x}($ Min-Max $)$ & $\bar{x}($ Min-Max $)$ & & $\bar{x}($ Min-Max $)$ & $\bar{x}($ Min-Max $)$ & \\
\hline \multirow[t]{2}{*}{ Orange } & 4.7 & 9.3 & $Z=-3.226$ & 4.7 & 9.3 & $Z=-1.078$ \\
\hline & $(0-9.3)$ & $(0-40.0)$ & $p=0.001^{*}$ & $(0-9.3)$ & $(0-40.0)$ & $p=0.281$ \\
\hline \multirow[t]{2}{*}{ Sour cherry } & 2.4 & 2.4 & $Z=-1.733$ & 1.4 & 2.4 & $Z=-2.534$ \\
\hline & $(0-4.8)$ & $(0-20.5)$ & $p=0.083$ & $(0-41.0)$ & $(0-4.8)$ & $p=0.011^{*}$ \\
\hline \multirow[t]{2}{*}{ Loquat } & 0.0 & 6.7 & $Z=-3.270$ & 0.0 & 6.7 & $Z=-2.058$ \\
\hline & $(0-6.7)$ & $(0-57.1)$ & $p=0.001^{*}$ & $(0-6.7)$ & $(0-13.3)$ & $p=0.040^{*}$ \\
\hline \multirow[t]{2}{*}{ Caffeinated coffee } & 17.1 & 34.3 & $Z=-1.186$ & 17.1 & 114.0 & $Z=-2.496$ \\
\hline & $(0-200.0)$ & $(0-200.0)$ & $p=0.236$ & $(0-171.4)$ & $(0-200.0)$ & $p=0.013^{*}$ \\
\hline \multirow[t]{2}{*}{ Coke } & 6.6 & 13.3 & $Z=-1.180$ & 0.0 & 57.1 & $Z=-3.257$ \\
\hline & $(0-228.0)$ & $(0-400.0)$ & $p=0.238$ & $(0-57.1)$ & $(0-200.0)$ & $p=0.001^{*}$ \\
\hline \multirow{2}{*}{ Diet coke } & 0.0 & 6.7 & $Z=-2.035$ & 0.0 & 0.0 & $Z=-1.089$ \\
\hline & $(0-6.7)$ & $(0-57.1)$ & $p=0.042^{*}$ & $(0-6.7)$ & $(0-200.0)$ & $p=0.276$ \\
\hline \multirow[t]{2}{*}{ Sparkling water } & 6.6 & 13.3 & $Z=-1.986$ & 6.6 & 6.7 & $Z=-0.343$ \\
\hline & $(0-200.0)$ & $(0-200.0)$ & $p=0.047^{*}$ & $(0-114.0)$ & $(0-200.0)$ & $p=0.731$ \\
\hline \multirow[t]{2}{*}{ Lemonade } & 0.0 & 6.7 & $Z=-3.210$ & 0.0 & 13.3 & $Z=-3.917$ \\
\hline & $(0-57.1)$ & $(0-200.0)$ & $p=0.001^{*}$ & $(0-13.3)$ & $(0-200.0)$ & $p=0.000^{* *}$ \\
\hline \multirow[t]{2}{*}{ Carot juice } & 0.0 & 6.7 & $Z=-2.053$ & 0.0 & 0.0 & $Z=-1.158$ \\
\hline & $(0-0)$ & $(0-57.1)$ & $p=0.040^{*}$ & $(0-6.6)$ & $(0-13.3)$ & $p=0.247$ \\
\hline \multirow[t]{2}{*}{ Orange juice } & 0.0 & 6.6 & $Z=-2.114$ & 0.0 & 6.6 & $Z=-2.520$ \\
\hline & $(0-13.3)$ & $(0-200.0)$ & $p=0.035^{*}$ & $(0-13.3)$ & $(0-200.0)$ & $p=0.012^{*}$ \\
\hline \multirow[t]{2}{*}{ Peach juice } & 0.0 & 0.0 & $Z=0.962$ & 0.0 & 6.6 & $Z=-2.762$ \\
\hline & $(0-57.1)$ & $(0-114.0)$ & $p=0.336$ & $(0-13.3)$ & $(0-200.0)$ & $p=0.006^{*}$ \\
\hline
\end{tabular}

Note: ${ }^{*} p<0.05$ and ${ }^{* *} p<0.001$, Mann-Whitney U test was used to compare the measurement values of two independent groups in non-parametric data.

$(p<0.05)$. The study also showed that male patients consume less pineapple, green almond, plum, loquat, date and orange than healthy males $(p<0.05)$. Similarly, male patients consume less diet coke, sparkling water, carot juice, orange juice and lemonade than healthy males $(p<0.05)$.

Fish consumption of female patients $(13.5 \pm 12.48 \mathrm{~g})$ was found to be lower than that of healthy group $(14.8 \pm 22.17 \mathrm{~g})(p<0.05)$. The study revealed that female patients consume less brussels sprouts, dill, mallow, artichoke, stinging nettle, savoy cabbage, celery, corn and chard than healthy females do $(p<0.05)$. Another result let us know that more curly lettuce is consumed by female patients $(p<0.05)$. Additionally, female patients consume less plum, cherry and loquat than healthy females $(p<0.05)$. Female patients consume less caffeinated coffee, orange juice, peach juice, coke and lemonade than healthy females do $(p<0.05)$ (Table 3 ).

Consumption of $2.5+$ portions of meat and meat products is higher in male patients, while consumption of $5+$ portions of fruits and vegetables and $2000+\mathrm{mL}$ of water is higher in healthy males. 
However, these results are not statistically significant ( $p>0.05)$. Similarly, there is not a statistically significant difference between dairy products, meat and meat products, fruit, vegetable and water consumption of female patients and healthy women ( $p>0.05$ ) (Table 4).

Eighty-eight point 9 percent of patients who have not suffered from stone recurrence, $100.0 \%$ of patients who have experienced stone recurrence once and twice and $92.9 \%$ of those who have experienced more than twice consume less than 3 portions of dairy products every day; $77.8 \%$ of

Table 4. Daily dietary water intake and portions of some food groups of individuals. Ankara, Turkey, 2018.

\begin{tabular}{|c|c|c|c|c|c|c|c|c|c|c|}
\hline \multirow{3}{*}{ Water/Food Groups } & \multicolumn{4}{|c|}{ Male } & \multirow{3}{*}{$\chi^{2 / p}$} & \multicolumn{4}{|c|}{ Female } & \multirow{3}{*}{$\chi^{2 / p}$} \\
\hline & \multicolumn{2}{|c|}{$\begin{array}{l}\text { Patient Group } \\
(n=29)\end{array}$} & \multicolumn{2}{|c|}{$\begin{array}{l}\text { Control Group } \\
\quad(n=29)\end{array}$} & & \multicolumn{2}{|c|}{$\begin{array}{l}\text { Patient Group } \\
\quad(n=15)\end{array}$} & \multicolumn{2}{|c|}{$\begin{array}{l}\text { Control Group } \\
\qquad(n=15)\end{array}$} & \\
\hline & $\mathrm{n}$ & $\%$ & $\mathrm{n}$ & $\%$ & & $n$ & $\%$ & $\mathrm{n}$ & $\%$ & \\
\hline \multicolumn{11}{|l|}{ Dairy products } \\
\hline$\geq 3$ portions/day & 1 & 3.4 & 1 & 3.4 & $\chi^{2}=0.000$ & 1 & 6.7 & 1 & 6.7 & $\chi^{2}=0.000$ \\
\hline$<3$ portions/day & 28 & 96.6 & 28 & 96.6 & $p=1.000$ & 14 & 93.3 & 14 & 93.3 & $p=1.000$ \\
\hline \multicolumn{11}{|c|}{ Meat and meat products } \\
\hline$\geq 2.5$ portions/day & 10 & 34.5 & 3 & 10.3 & $\chi^{2}=3.569$ & 1 & 6.7 & 1 & 6.7 & $\chi^{2}=0.000$ \\
\hline$<2.5$ portions/day & 19 & 65.5 & 26 & 89.7 & $p=0.059$ & 14 & 93.3 & 14 & 93.3 & $p=1.000$ \\
\hline \multicolumn{11}{|l|}{ Vegetable and fruit } \\
\hline$\geq 5$ portions/day & 15 & 51.7 & 17 & 58.6 & $\chi^{2}=0.279$ & 7 & 46.7 & 6 & 40.0 & $\chi^{2}=0.136$ \\
\hline$<5$ portions/day & 14 & 48.3 & 12 & 41.4 & $p=0.597$ & 8 & 53.3 & 9 & 60.0 & $p=0.713$ \\
\hline \multicolumn{11}{|l|}{ Water } \\
\hline$\geq 2000 \mathrm{~mL}$ day & 7 & 24.1 & 15 & 51.7 & $\chi^{2}=3.588$ & 5 & 33.3 & 9 & 60.0 & $\chi^{2}=1.205$ \\
\hline$<2000 \mathrm{~mL}$ day & 22 & 75.9 & 14 & 48.3 & $p=0.058$ & 10 & 66.7 & 6 & 40.0 & $p=0.272$ \\
\hline
\end{tabular}

Note: Chi-square test was applied.

Table 5. Daily dietary water intake account and consumption portions of some food groups according to stone formation recurrence of individuals. Ankara, Turkey, 2018.

\begin{tabular}{|c|c|c|c|c|c|c|c|c|c|}
\hline \multirow{2}{*}{ Water/Food Groups } & \multicolumn{2}{|c|}{0} & \multicolumn{2}{|c|}{1} & \multicolumn{2}{|c|}{2} & \multicolumn{2}{|c|}{$>2$} & \multirow{2}{*}{$\chi^{2} / p$} \\
\hline & $n$ & $\%$ & $n$ & $\%$ & $\mathrm{n}$ & $\%$ & $\mathrm{n}$ & $\%$ & \\
\hline \multicolumn{10}{|l|}{ Dairy products } \\
\hline$\geq 3$ portions/day & 1 & 11.1 & 0 & 0.0 & 0 & 0.0 & 1 & 7.1 & $\chi^{2}=2.112$ \\
\hline$<3$ portions/day & 8 & 88.9 & 10 & 100.0 & 11 & 100.0 & 13 & 92.9 & $p=0.550$ \\
\hline \multicolumn{10}{|c|}{ Meat and meat products } \\
\hline$\geq 2.5$ portions/day & 2 & 22.2 & 2 & 20.0 & 4 & 36.4 & 3 & 21.4 & $\chi^{2}=1.023$ \\
\hline$<2.5$ portions/day & 7 & 77.8 & 8 & 80.0 & 7 & 63.6 & 11 & 78.6 & $p=0.796$ \\
\hline \multicolumn{10}{|l|}{ Vegetable and fruit } \\
\hline$\geq 5$ portions/day & 7 & 77.8 & 4 & 40.0 & 4 & 36.4 & 7 & 50.0 & $\chi^{2}=3.996$ \\
\hline$<5$ portions/day & 2 & 22.2 & 6 & 60.0 & 7 & 63.6 & 7 & 50.0 & $p=0.262$ \\
\hline \multicolumn{10}{|l|}{ Water } \\
\hline$\geq 2000 \mathrm{~mL} /$ day & 3 & 33.3 & 4 & 40.0 & 2 & 18.2 & 3 & 21.4 & $\chi^{2}=1.683$ \\
\hline$<2000 \mathrm{~mL} /$ day & 6 & 66.7 & 6 & 60.0 & 9 & 91.8 & 11 & 78.6 & $p=0.641$ \\
\hline
\end{tabular}

Note: Chi-square test was applied 
patients with no stone recurrence consume more than 5 portions of fruit and vegetable every day, while $50.0 \%$ of patients with more than two recurrences do so. Moreover, $66.7 \%$ of patients with no stone recurrence consume less than $2000 \mathrm{~mL}$ of water every day and $91.8 \%$ of those with two recurrences do not exceed that limit. However, there is not a statistically significant difference between number of stone recurrence and consumption of food and food groups included in the Table 5 ( $p>0.05)$.

\section{DISCUSSION}

Salt consumption is among the important factors of kidney stone formation [17]. Nouvenne et al. [18] found out in a study that a diet with less amount of salt reduces kidney stone formation risk by decreasing sodium and calcium excretion $(p<0.001)$. In addition, hypervolemia resulting from increased dietary salt consumption increases urinary sodium excretion by reducing reabsorption of sodium through kidney tubules [19]. However, a high throughput of sodium is not all bad in terms of stone risk since a high intake of salt is likely to stimulate the thirst response and make the patient drink more. Nevertheless, a high salt diet is one of the scourges of modern society for many other reasons [17]. This study showed that both genders in patient group consume more salt in their food than healthy individuals do $(p<0.05)$. Moreover, habit of salting without tasting is more frequent in male patients than healthy males $(p<0.05)$ (Table 1$)$. From this fact, it is considered that high salt consumption habit of patient group increases kidney stone formation risk by boosting urinary sodium and calcium excretion $[17,19]$.

It is reported that fluid intake decreases stone formation risk by diluting urine $[20,21]$. According to study results, daily fluid intake of both genders was found to be lower than healthy individuals $(p<0.05)$ (Table 1$)$. This result is a clue that kidney stone recurrence risk may be high in the patient group. Studies in the literature suggest that increased fluid intake decreases calcium phosphate, calcium oxalate and monosodium urate concentration, and thus reduces stone formation risk [20].

Positive family history is one of the most important risk factors of nephrolithiasis [22]. In a study conducted for evaluation of correlation between nephrolithiasis and family history, it was found out that $37.5 \%$ of male patients and $27.0 \%$ of female patients had positive family history [23]. Supporting above-mentioned results, this study showed that $51.7 \%$ of male patients and $53.3 \%$ of female patients have kidney stone history in their family (Table 2). Genetic susceptibility is an important risk factor in kidney stone formation like many other chronic diseases [24].

Nephrolithiasis is a highly recurring disease and prevention of stone recurrence during treatment process is as important as the treatment itself $[25,26]$. The study showed that $79.3 \%$ of male patients and $80.0 \%$ of female patients have kidney stone formation recurrence (Table 2 ). High stone recurrence rates are considered to be resulted from inefficient treatment processes.

Traditional and alternative medicine applications have been widely employed from past to present [27]. The study showed that patients with nephrolithiasis consume olive oil, stinging nettle, parsley juice, highbush cranberry juice, lemon juice, kefir and oleaster juice in order to pass the kidney stone (Table 2). As the study was conducted nationwide, the foods and beverages consumed to pass kidney stone may vary from region to region. Recent studies in the literature report that various foods and drinks may have anti-lithogenic $[10,28]$. In order to use anti-lithogenic foods and drinks in the 
treatment of kidney stone disease further human studies should be carried out, their mechanisms should be examined thoroughly, and correct and effective dose should be determined.

Containing high amount of calcium, dairy products reduce stone formation risk by decreasing intestinal absorption of oxalate [29]. However, there are several studies which suggest that there is not a correlation between dietary calcium intake and stone formation [30,31]. In a study carried out by Taylor \& Curhan [32], it was found out that increased dairy products consumption reduces stone formation risk. However, in this study, there is not a gender-based difference between dairy products consumption of patient group and healthy individuals ( $p>0.05$ ) (Table 3 ). Additionally, $88.9 \%$ of patients who have not suffered from stone recurrence, $100.0 \%$ of patients who have experienced stone recurrence once and twice and $92.9 \%$ of those who have experienced more than twice consume less than 3 portions of dairy products every day. However, there is also not a statistically significant difference between number of recurrence and dairy products consumption ( $p>0.05$ ) (Table 5). Therefore, it is recommended to consume calcium as required rather than limiting dairy products to prevent stone formation [33].

Consumption of animal and vegetable protein in adequate amounts is quite important for health, as well [34]. Nonetheless, it is reported that increased animal protein intake causes an increase in acid production through methionine and cysteine sulphuric acid metabolism [9]. As a result of acid load increase, urinary calcium excretion rises as calcium breakdown scales up and calcium reabsorption decreases in kidneys [9,35]. Moreover, high amount of fat contained in animal protein sources may increase intestinal oxalate absorption says some studies [36]. In this study, meat consumption of male patients was found to be higher than healthy males $(p<0.05)$ (Table 3$)$. In line with this data, it is fair to suggest that high meat consumption may increase stone formation risk as it steps up acid production and fat intake (g) $[9,35,36]$.

It is known that fatty acids in fish have important functions regarding human health [37]. For example, it is reported that $\mathrm{n}-3$ fatty acids contained in fish and fish oil may reduce kidney stone formation risk [38]. Some studies have proved that fish oil supplement in the patients with idiopathic calcium oxalate stone reduces urinary oxalate and calcium excretion $[39,40]$. Additionally, $n-3$ fat acid supplement shows anti-lithogenic effecy by suppressing prostoglandin E2 production which increases intestinal calcium absorption [40]. In this study, fish consumption of female patients was found to be lower than healthy females $(p<0.05$ ) (Table 3 ). It can be concluded that increased $n-3$ fatty acid intake through high amount of fish consumption in healthy females may reduce kidney stone formation risk by decreasing urinary oxalate and calcium excretion $[39,40]$.

Some studies indicate that fruit and vegetable consumption creates anti-lithogenic effect by reducing calcium excretion and increasing urinary citrate excretion, while some other studies suggest that such effect is caused by phenolic compounds $[8,10,41]$. In a study carried out by Sorensen et al. [10] in 2014, it was found out that individuals with kidney stone disease consumed less amount of fruit and vegetable than healthy individuals did and the difference was statistically significant $(p<0.05)$. Similarly, this study demonstrates that male and female patients consume less amount of fruit and vegetable than healthy individuals (Table 3). Thanks to consumption of fruits and vegetables in high amounts, healthy individuals intake more macro and micro-nutrients such as pulp, phenolic compounds, citrate and potassium which are known to have anti-lithogenic effect. Despite such benefits, some vegetables such as spinach, beet, chard, tea, chocolate and nuts which are rich in oxalate may increase stone formation risk [42]. Therefore, it can be recommended for patients with kidney stone not to consume high amounts of vegetables known to be rich in oxalate. 
Besides increasing total fluid intake, beverages take important roles in kidney stone formation risk due to their caffeine, alcohol, oxalate, potassium, citrate and similar other content $[28,38,43]$. In a study carried out by Ferraro et al. [43], it was found out that increased caffeine consumption is correlated with stone formation risk. Another study proved that sugar-free sparkling water, coke and orange juice consumption reduces kidney stone formation risk $(p<0.05)$ [28]. In addition, numerous studies put forward that increased orange juice and lemonade consumption reduces stone formation risk by scaling up urinary citrate excretion $[44,45]$. It was found out in this study that male patients consume less diet coke, sparkling water, carrot juice, orange juice and lemonade than healthy individuals $(p<0.05)$. Similarly, female patients consume less caffeinated coffee, orange juice, peach juice, coke and lemonade than healthy females $(p<0.05)$ (Table 3 ). In the light of this data, less consumption of above stated beverages by patient group in both genders can be considered as a risk factor in terms of stone formation. These beverages include coke which is not recommended due to its adverse effects on human health. However, it can be considered that coke suppresses stone formation risk thanks to its caffeine content.

The study showed that consumption of $2.5+$ portions of meat products is higher in male patients, while consumption of $5+$ portions of fruit and vegetable is lower. However, these results are not statistically significant ( $p>0.05$ ) (Table 4 ). Dietary protein intake is one of the most important causes of change in Potential Renal Acid Load (PRAL) value [46]. Protein intake from animal origin mainly leads to the production of acid through methionine and cysteine sulfuric acid metabolism [9]. Additionally, consumption of $2000+\mathrm{mL}$ of water is lower in female patients than healthy females. These results are not statistically significant, as well ( $p>0.05$ ) (Table 4). The fact that individuals in the patient group consume more meat and meat products and less fruit, vegetable and water may boost kidney stone formation risk.

It is reported that increased water, fruit and vegetable consumption decreases kidney stone formation and recurrence risk $[10,47]$. Taylor et al. [48] found out that fruit and vegetable consumption reduces stone formation and recurrence. This study demonstrated that consumption of 5+ portions of fruit and vegetable and $2000+\mathrm{mL}$ of water is higher in patients with no recurrence. However, there is not a statistically significant difference between number of stone recurrence and fruit, vegetable and water consumption ( $p>0.05$ ) (Table 5 ). This result is a clue that citrate, magnesium and potassium intake due to fruit and vegetable consumption and increased urine volume due to water intake can reduce stone recurrence risk.

\section{CONCLUSION}

As a consequence, it can be suggested that general eating habits such as dietary total fluid intake, salt consumption habits, fruit and vegetable consumption, meat and meat products consumption can effect kidney stone formation and recurrence. Therefore, a personal diet plan should be prepared and prescribed for each individual with nephrolithiasis.

\section{CONTRIBUTORS}

MA ICER idealized and developed the project studied, collected the data, interpreted and analyzed the data, reviewed the literature and wrote the article. M GEZMEN-KARADAG supervised the development of the project, reviewed the article and approved its final version. 


\section{REFERENCES}

1. Shadman A, Bastani B. Kidney calculi: Pathophysiology and as a systemic disorder. Iran J Kidney Dis. 2017;11(3):180-91.

2. Worcester EM, Coe FL. Nephrolithiasis. Prim Care. 2008;35(2):369-91.

3. Ziemba JB, Matlaga BR. Epidemiology and economics of nephrolithiasis. Investig Clin Urol. 2017;58(5):299-306.

4. Khan MS, Lari QH, Khan MA. Unani concept of nephrolithiasis (hisat-e-kulyah) and its management: An overview. WJPMR. 2016;2(5):247-52.

5. Shin S, Srivastava A, Alli NA, Bandyopadhyay BC. Confounding risk factors and preventative measures driving nephrolithiasis global makeup. World J Nephrol. 2018;7(7):129-42.

6. Dissayabutra T, Kalpongkul N, Rattanaphan J, Boonla C, Srisa-art M, Ungjaroenwathana W, et al. Urinary stone risk factors in the descendants of patients with kidney stone disease. Pediatr Nephrol. 2018;33(7):1173-81.

7. Mangano KM, Walsh SJ, Kenny AM, Insogna KL, Kerstetter JE. Dietary acid load is associated with lower bone mineral density in men with low intake of dietary calcium. J Bone Miner Res. 2014;29(2):500-6.

8. Penczynski KJ, Krupp D, Bring A, Bolzenius K, Remer T, Buyken AE. Relative validation of 24-h urinary hippuric acid excretion as a biomarker for dietary flavonoid intake from fruit and vegetables in healthy adolescents. Eur J Nutr. 2017;56(2):757-66.

9. Vezzoli G, Dogliotti E, Terranegra A, Arcidiacono T, Macrina L, Tavecchia M, et al. Dietary style and acid load in an Italian population of calcium kidney stone formers. Nutr Metab Cardiovasc Dis. 2015;25(6):588-93.

10. Sorensen MD, Hsi RS, Chi T, Shara N, Wactawski-Wende J, Kahn AJ, et al. Dietary intake of fiber, fruit and vegetables decreases the risk of incident kidney stones in women: a Women's Health Initiative report. J Urol. 2014;192(6):1694-9.

11. Guerra A, Folesani G, Mena P, Ticinesi A, Allegri F, Nouvenne A, et al. Hippuric acid in 24 h urine collections as a biomarker of fruits and vegetables intake in kidney stone formers. Int J Food Sci Nutr. 2014;65(8):1033-8.

12. Mirzaei K, Aghamir SMK, Modaresi SS, Yekaninejad MS. Major Dietary Patterns and kidney stone formation among Iranian men. J Nutr Sci Diet. 2017;3(3):11-7.

13. Icer MA, Gezmen-Karadag M, Sozen S. Can urine osteopontin levels, which may be correlated with nutrition intake and body composition, be used as a new biomarker in the diagnosis of nephrolithiasis? Clin Biochem. 2018;60:38-43.

14. Fakhoury MQ, Gordon B, Shorter B, Renson A, Borofsky MS, Cohn MR, et al. Perceptions of dietary factors promoting and preventing nephrolithiasis: A cross-sectional survey. World J Urol. 2018:1-9. Epud 2018 Dec 15.

15. Icer MA, Gezmen-Karadag M. Determination of the effect of nutritional status on PRAL level in patients with nephrolithiasis. Gümüşhane Univ J Health Sci. 2018; 7(4): 1-9

16. Seeger $H$, Kaelin A, Ferraro PM, Weber $D$, Jaeger $P, A m b u e h l ~ P$, et al. Changes in urinary risk profile after short-term low sodium and low calcium. 2017;18(1):349.

17. Robertson W. Dietary recommendations and treatment of patients with recurrent idiopathic calcium stone disease. Urolithiasis. 2016;44(1):9-26.

18. Nouvenne A, Meschi T, Prati B, Guerra A, Allegri F, Vezzoli G, et al. Effects of a low-salt diet on idiopathic hypercalciuria in calcium-oxalate stone formers: A 3-mo randomized controlled trial. Am J Clin Nutr. 2010;91(3):565-70.

19. Afsar B, Kiremit MC, Sag AA, Tarim K, Acar O, Esen T, et al. The role of sodium intake in nephrolithiasis: Epidemiology, pathogenesis, and future directions. Eur J Intern Med. 2016;35:16-9.

20. Fink HA, Akornor JW, Garimella PS, MacDonald R, Cutting A, Rutks IR, et al. Diet, fluid, or supplements for secondary prevention of nephrolithiasis: A systematic review and meta-analysis of randomized trials. Eur Urol. 2009;56(1):72-80.

21. Xu C, Zhang C, Wang X-L, Liu T-Z, Zeng X-T, Li S, et al. Self-fluid management in prevention of kidney stones: A PRISMA-compliant systematic review and dose-response meta-analysis of observational studies. Medicine. 2015;94(27):e1042. 
22. Guerra A, Folesani G, Nouvenne A, Ticinesi A, Allegri F, Pinelli S, et al. Family history influences clinical course of idiopathic calcium nephrolithiasis: Case-control study of a large cohort of Italian patients. J Nephrol. 2016;29(5):645-51.

23. Jabbar F, Asif M, Dutani H, Hussain A, Malik A, Kamal MA, et al. Assessment of the role of general, biochemical and family history characteristics in kidney stone formation. Saudi J Biol Sci. 2015;22(1):65-8.

24. Zeng G, Mai Z, Xia S, Wang Z, Zhang K, Wang L, et al. Prevalence of kidney stones in China: An ultrasonography based cross-sectional study. BJU Int. 2017;120(1):109-16.

25. Chidambaram A, Rodriguez D, Khan S, Gower L. Biomimetic Randall's plaque as an in vitro model system for studying the role of acidic biopolymers in idiopathic stone formation. Urolithiasis. 2015;43(1):77-92.

26. Denu-Ciocca C. Medical management of hypocitraturia. In Penniston K, Goldfarb DS, editors. Pocket guide to kidney stone prevention. New York: Springer; 2015. p.55-61.

27. Yuan $\mathrm{H}, \mathrm{Ma}$ Q, Ye L, Piao G. The traditional medicine and modern medicine from natural products. Molecules. 2016;21(5):559.

28. Ferraro PM, Taylor EN, Gambaro G, Curhan GC. Soda and other beverages and the risk of kidney stones. Clin J Am Soc Nephrol. 2013;8(8):1389-95.

29. Holmes RP, Knight J, Assimos DG. Lowering urinary oxalate excretion to decrease calcium oxalate stone disease. Urolithiasis. 2016;44(1):27-32.

30. Fellström B, Danielson B, Karlström B, Lithell H, Ljunghall S, Vessby B. Dietary habits in renal stone patients compared with healthy subjects. BJU Int. 1989;63(6):575-80.

31. Trinchieri A, Mandressi A, Luongo P, Longo G, Pisani E. The influence of diet on urinary risk factors for stones in healthy subjects and idiopathic renal calcium stone formers. BJU Int. 1991;67(3):230-6.

32. Taylor EN, Curhan GC. Dietary calcium from dairy and nondairy sources, and risk of symptomatic kidney stones. J Urol. 2013;190(4):1255-9.

33. Sorensen MD. Calcium intake and urinary stone disease. Transl Androl Urol. 2014;3(3):235-40.

34. Wu G. Dietary protein intake and human health. Food Fuct. 2016;7(3):1251-65.

35. Ferraro PM, Mandel El, Curhan GC, Gambaro G, Taylor EN. Dietary protein and potassium, diet-dependent net acid load, and risk of incident kidney stones. Clin J Am Soc Nephrol. 2016;11(10):1834-44.

36. Paßlack N, Burmeier H, Brenten T, Neumann K, Zentek J. Relevance of dietary protein concentration and quality as risk factors for the formation of calcium oxalate stones in cats. J Nutr Sci. 2014;3(51):1-10.

37. Golden CD, Allison EH, Cheung WW, Dey MM, Halpern BS, McCauley DJ, et al. Nutrition: Fall in fish catch threatens human health. Nature. 2016;534(7607):317-20.

38. Gul Z, Monga M. Medical and dietary therapy for kidney stone prevention. Korean J Urol. 2014;55(12):775-9.

39. Buck AC, Davies R, Harrison T. The protective role of eicosapentaenoic acid [EPA] in the pathogenesis of nephrolithiasis. J Urol. 1991;146(1):188-94.

40. Baggio B, Gambaro G, Zambon S, Marchini F, Bassi A, Bordin L, et al. Anomalous phospholipid n-6 polyunsaturated fatty acid composition in idiopathic calcium nephrolithiasis. JASN. 1996;7(4):613-20.

41. Nouvenne A, Ticinesi A, Morelli I, Guida L, Borghi L, Meschi T. Fad diets and their effect on urinary stone formation. Transl Androl Urol. 2014;3(3):303-12.

42. Bihl G, Meyers A. Recurrent renal stone disease-advances in pathogenesis and clinical management. Lancet. 2001;358(9282):651-6.

43. Ferraro PM, Taylor EN, Gambaro G, Curhan GC. Caffeine intake and the risk of kidney stones. Am J Clin Nutr. 2014;100(6):1596-603.

44. Odvina CV. Comparative value of orange juice versus lemonade in reducing stone-forming risk. Clin J Am Soc Nephrol. 2006;1(6):1269-74.

45. Gambaro G, Trinchieri A. Recent advances in managing and understanding nephrolithiasis/nephrocalcinosis. F1000Research. 2016;5:1-8. 
46. Teunissen-Beekman KF, Dopheide J, Geleijnse JM, Bakker SJ, Brink EJ, Leeuw PW, et al. Effect of increased protein intake on renal acid load and renal hemodynamic responses. Physiol Rep. 2016;4(5):e12687.http// dx.doi.org/10.14814/phy2.12687

47. Prezioso D, Strazzullo P, Lotti T, Bianchi G, Borghi L, Caione $P$, et al. Dietary treatment of urinary risk factors for renal stone formation: A review of CLU working group. Arch Ital Urol Androl. 2015;87(2):105-20.

48. Taylor EN, Stampfer MJ, Mount DB, Curhan GC. DASH-style diet and 24-hour urine composition. Clin J Am Soc Nephrol. 2010;5(12):2315-22.

Received: February 19, 2019

Final version: May 9, 2019

Approved: May 23, 2019 